\title{
APTITUDE AU VÊLAGE ET CONDITIONS DE MISE BAS DES GÉNISSES CULARDES CHAROLAISES ET BLONDES D'AQUITAINE
}

\author{
F. Ménissier et G.P. Fabre. - Station de Génétique quantitative et appliquée, C. N. R. Z., \\ I. N. R. A., 78-Jouy-en-Josas (France).
}

Nous avons étudié les conditions de mise bas de $\mathrm{I}_{3}$ génisses charolaises ( $\mathrm{CH}$ ) et $\mathrm{I}_{4}$ blonde d'Aquitaine $(B A)$ présentant le caractère culard et accouplées suivant un plan factoriel à un taureau culard de chacune de ces 2 races.

Les pourcentages de vêlages difficiles (césariennes et extractions forcées) et de césariennes seules étaient respectivement de :

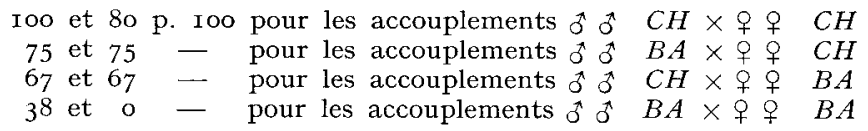

La fréquence élevée de difficultés de vêlages associée à l'emploi de géniteurs charolais s'explique par des différences morphologiques liées à la fois au volume du veau (effect direct) et à l'ouverture pelvienne des mères (effet maternel). Le pourcentage supérieur de vêlages difficiles correspondant à l'emploi de taureaux charolais ( +27 p. I0o) s'explique essentiellement par la supériorité de poids de naissance des veaux issus de ces taureaux $(+\mathrm{I} 0,7$ et 8,0$)$ respectivement sur génisses $C H$ et $B A$ ). Par contre, le pourcentage plus élevé de difficultés de vêlage avec les femelles $C H$ par rapport aux $B A(+33 \mathrm{p}$. Ioo et $+37 \mathrm{p}$. Ioo) avec les races paternelles respectivement, ne correspond pas à un accroissement de poids à la naissance $(+2,2$ et $-0,4 \mathrm{~kg}$ ) des veaux issus de vaches charolaises dont les poids au vêlage sont pourtant supérieurs à ceux des vaches blondes $(+46$ et $+29 \mathrm{~kg}$ suivant les races paternelles). L'accroissement du taux de vêlage difficiles chez les femelles $C H$ est en fait imputable à la réduction de leur ouverture pelvienne : $-\mathrm{I} 5 \mathrm{~cm}$ à 21 mois et $-22 \mathrm{~cm}$ après vêlage à 3 ans.

Ces résultats reflètent le double processus d'accroissement du poids à la naissance et de réduction de l'ouverture pelvienne qui accompagne l'augmentation de la musculature et de la compacité plus développées en race charolaise qu'en race blonde d'Aquitaine avec des animaux de type culard. La sélection de bovins culards en race blonde d'Aquitaine ne présente pas de ce fait les inconvénients que l'on observe en Chavolais du point de vue de l'aptitude au vêlage.

\section{NOTE SUR L'ÉVOLUTION DE LA RACE CHAROLAISE SOUS L'EFFET DE LA SÉLECTION}

B. Vissac, H. A. Molinuevo et F. Ménissier, - Station de Génétique quantitative et appliquée, C. N.R.A., I. N.R. A., 78-Jouy-en-Josas (France).

Depuis le début du siècle la race charolaise a été utilisée puis sélectionnée en dehors de sa zone d'origine du centre de la France (type " $\mathrm{N}$ ") dans des optiques diverses. En Amérique (Brésil et Texas), on a recherché un type d'animal adapté à l'élevage extensif (type "A 》) dans des zones subtropicales; dans le sud de la France où le développement de l'insémination artificielle dans les petits élevages a permis l'emploi de mâles charolais, on utilise surtout des taureaux à forte musculature (" $M$ 》) voire depuis quelques années des culards (" $C$ ").

Grâce aux diverses expériences comparatives de la descendance de ces différents types de taureaux (désignés par les lettres entre parenthèses) accouplés avec un même cheptel femelle, nous avons tenté d'estimer leur valeur génétique actuelle respective pour le poids à la naissance considéré comme le critère de croissance le plus héritable. Nous avons utilisé les résultats des essais effectués au Canada d'une part (A-N) et en France d'autre part (C-M). Dans la mesure où nous admettons que le poids à la naissance a un déterminisme génétique de type additif et que les taureanx limousins utilisés en race pure et en croisement sont de même type, 
la comparaison des écarts de poids des veaux charolais ("N") et limousins exploité en zone de race pure d'une part et de ceux des veaux croisés issus de taureaux charolais (" $\mathbf{M}$ ") et limousins utilisés sur le même cheptel femelle (sud-ouest de la France) d'autre part, permet d'estimer la différence de valeur génétique (M-N) entre ces deux types de Charolais.

Ces différences concordent aussi bien avec d'autres expériences françaises réalisées sur bovins normaux et culards, qu'avec les variations, constatées dans divers pays américains selon l'origine des taureaux charolais, de la supériorité des descendants de mâles charolais sur celles des taureaux Hereford ou Aberdeen-Angus.

Tous ces résultats traduisent l'évolution du potentiel de croissance sous l'effet de la sélection naturelle (conditions d'élevage) et artificielle (recherche de la musculature). L'écart minimun de poids à la naissance (20 p. IOO) paraissant supérieur à celui entre les poids adultes des types extrêmes, cela tendrait à indiquer qu'il y a modification de la forme de la courbe de croissance avec l'augmentation de la musculature.

\title{
L'INTERFÉRENCE DU CARACTÈRE “CULARD » ETT DE LA SÉLECTION BASÉE SUR LA CONFORMATION, DANS LA RACE BOVINE DE MOYENNE ET HAUTE-BELGIQUE
}

\author{
R. Hanset. -- Chaire de Génétique, Faculté de Médecine vétérinaive, Université de Liège, \\ Cureghem-Bruxelles (Belgique).
}

En race de Moyenne et Haute-Belgique, se poursuit une sélection en faveur d'une conformation caractérisée par l'arrière-main et la ligne du dessus fortement musclées, la croupe inclinée, les hanches effacées, la côte arrondie. L'auteur se livre à un bilan de cette sélection, du point de vue du caractère culard, dans deux provinces représentatives. De I958 à I970, la fréquence du caractère culard, dans la descendance de l'insémination artificielle, est passée de 2,28 p. Ioo à 9,I p. Ioo dans une province, de I,43 p. Ioo à $7,13 \mathrm{p}$. I oo dans la seconde. La fréquence des césariennes est de l'ordre de i i p. Ioo dans les deux provinces. Si on caractérise chaque taureau IA par la proportion de ses descendants qui présentent le caractère culard, on s'aperçoit qu'il existe une grande hétérogénéité entre taureaux. Un déterminisme monogénique pour le caractère culard est compatible avec les données d'observation à la condition d'admettre l'intervention de gènes modificateurs (au moins une paire) à grande amplitude d'action. L'évolution de la conformation, dans la descendance normale des mêmes taureaux IA est étudiée parallèlement à l'évolution de la fréquence du caractère culard. La relation entre ces deux événements est faible. Dans la population considérée, le résultat de la sélection basée sur la conformation aurait été d'accroître la fréquence du gène culard, d'où la fréquence du caractère, sans pour autant avoir amélioré d'une façon sensible le développement moyen de la musculature des animaux non-culards.

\section{Essai DE CARActÉRISATION BIOChIMIQUE DU MUSCLE DE BOVINS CULARDS (1)}

\author{
M. Ansay. - Chaire de Génétique, Faculté de Médecine vétérinaire, Université de Liège, \\ Cureghem-Bruxelles. (Belgique).
}

La teneur en ADNP du muscle demi-tendineux chez des animaux culards et normaux a été examinée depuis le troisième mois de la vie fœetale jusqu'à l'âge adulte. La concentration en ADNP reste élevée (20 à $25 \mathrm{mg}$ d'ADNP) du troisième au cinquième mois environ de la: vie fotale, puis diminue progressivement pour passer à Io $\mathrm{mg}$ environ à la naissance, à $2 \mathrm{mg}$ à l'âge de 15 mois. Pendant toute la période envisagée, les animaux culards ont une concentration équivalente en ADNP.

(1) Travail exécuté sous les auspices de l'Institut pour l'encouragement de la Recherche scientifique dans l'Industrie et l'Agriculture. 